\title{
Regulation of Steroid Hormone Receptor Function By the 52-kDa FK506-Binding Protein (FKBP52)
}

\author{
Jeffrey C. Sivils ${ }^{1}$, Cheryl L. Storer ${ }^{1}$, Mario D. Galigniana ${ }^{2}$, and Marc B. Cox ${ }^{1}$ \\ ${ }^{1}$ The Border Biomedical Research Center and Department of Biological Sciences, University of \\ Texas at EI Paso, EI Paso, TX, USA \\ ${ }^{2}$ Instituto de Biología y Medicina Experimental (IBYME)-CONICET Departamento de Química \\ Biológica, Facultad de Ciencias Exactas y Naturales Universidad de Buenos Aires, Buenos Aires, \\ Argentina
}

\begin{abstract}
The large FK506-binding protein FKBP52 has been characterized as an important positive regulator of androgen, glucocorticoid and progesterone receptor signaling pathways. FKBP52 associates with receptor-Hsp90 complexes and is proposed to have roles in both receptor hormone binding and receptor subcellular localization. Data from biochemical and cellular studies has been corroborated in whole animal models as fkbp52-deficient male and female mice display characteristics of androgen, glucocorticoid and/or progesterone insensitivity. FKBP52 receptor specificity and the specific phenotypes displayed by the $f k b p 52$-deficient mice have firmly established FKBP52 as a promising target for the treatment of a variety of hormone-dependent diseases. Recent studies demonstrated that the FKBP52 FK1 domain and the proline-rich loop within this domain are functionally important for FKBP52 regulation of receptor function. Based on these data, efforts are currently underway to target the FKBP52 FK1 domain and the prolinerich loop with small molecule inhibitors.
\end{abstract}

\section{Introduction}

In the absence of ligand, steroid receptors remain sequestered in the cytoplasm and/or nucleus in complex with chaperone and co-chaperone proteins including, but not limited to heat-shock protein 90 (Hsp90), heat-shock protein 70 (Hsp70), a 23-kDa co-chaperone (p23) and one of a family of proteins characterized by the presence of an Hsp90-binding tetratricopeptide repeat (TPR) domain (also termed immunophilins) [reviewed in 1]. The large 52-kDa FK506-binding protein (FKBP52; also termed FKBP4) has been shown to associate with steroid receptor complexes in vitro and acts as a specific positive regulator of androgen (AR), glucocorticoid (GR) and progesterone receptor (PR) function [2-4]. Despite the significant sequence, structural and/or functional homology that exists between some steroid hormone receptors (e.g. GR and MR) FKBP52 does not functionally affect any other known Hsp90 client proteins including the mineralocorticoid (MR) and estrogen receptors (ER). Thus, FKBP52 specifically regulates a small subset of Hsp90 client proteins and a small subset of steroid hormone receptors [5]. This specificity and the specific phenotypes

\footnotetext{
(C) 2011 Elsevier Ltd. All rights reserved.

Corresponding Author: Cox, Marc B. (mbcox@utep.edu).

Publisher's Disclaimer: This is a PDF file of an unedited manuscript that has been accepted for publication. As a service to our customers we are providing this early version of the manuscript. The manuscript will undergo copyediting, typesetting, and review of the resulting proof before it is published in its final citable form. Please note that during the production process errors may be discovered which could affect the content, and all legal disclaimers that apply to the journal pertain.
} 
observed in the fkbp52-deficient mice establish FKBP52 as an attractive therapeutic target for the treatment of any disease that depends upon a functional AR, GR and/or PR signaling pathway. FKBP52 association with receptor-chaperone complexes results in an enhancement of receptor hormone binding $[3,6,7]$ and influences receptor localization within the cell [reviewed in 8]. The exact mechanism(s) of FKBP52 action is currently unknown, but important progress has recently been made. Two partial crystal structures that, together, span the full-length protein have been solved [9]. FKBP52 consists of a C-terminal Hsp90-binding TPR domain, an N-terminal FK1 domain that contains a functional peptidyl/ prolyl isomerase (PPIase) active site to which the immunosuppressive ligand FK506 binds, and a middle FK2 domain that is similar to FK1 but lacks PPIase activity (Figure 1). In this review we discuss the most significant findings over the last seven years including roles for FKBP52 in hormone-dependent physiological processes, FKBP52 regulation of receptor nuclear localization, and functional domain mapping studies to identify critically important functional residues, regions and/or domains on FKBP52. Finally, based on the functional domain mapping studies, we discuss possible therapeutic targeting strategies.

\section{Physiological Roles}

Based on previous biochemical and cellular studies one would predict that $f k b p 52$-deficient mice would display phenotypes consistent with various hormone insensitivity syndromes. Indeed, fkbp52-deficient male mice display phenotypes consistent with partial androgen insensitivity syndrome including dysgenic seminal vesicles and prostate, ambiguous external genitalia, hypospadias and nipples retained into adulthood $[2,10]$. In addition to alterations in primary and accessory sex organs, the epididymis of $f k b p 52$-deficient male mice have significantly depressed sperm counts and the sperm display abnormal morphology [11]. Interestingly, in this same study FKBP52 was also reported to be present in epididymal sperm flagella, suggesting that FKBP52 association with dynein motor proteins (discussed below) may be involved in flagellar movement, which is an androgen-independent process.

The loss of FKBP52 in female $f k b p 52$-deficient mice results in infertility in both the C57BL6/129 and CD1 backgrounds [4,12]. The mice appear morphologically normal; moreover, ovulation and fertilization are not overtly hindered. Infertility appears to be due to progesterone resistance and uterine defects resulting in embryonic implantation and decidualization failure $[12,13]$. Implantation failure as a result of reduced progesterone signaling may be due to increased uterine oxidative stress in $f k b p 52$-deficient female mice as a result of reduced levels of the anti-oxidant peroxiredoxin-6 (PRDX6) [14]. Implantation failure was observed in $f k b p 52$-deficient, but not wild-type female mice with paraquatinduced oxidative stress [12]. Exogenous progesterone alone did not rescue implantation, but it did with the addition of an antioxidant. Finally, the loss of FKBP52 promotes the onset of endometriosis as progesterone resistance in $f k b p 52$-deficient mice manifests with increased cell proliferation, inflammation, and angiogenesis resulting in endometriotic lesions [15]. Furthermore, women with endometriosis have reduced FKBP52 expression compared to women without endometriosis.

FKBP52 knockout also results in phenotypes related to GR signaling. Homozygous fkbp52deficient mice were initially reported to have high serum corticosterone levels, which were hypothesized to result from the mice compensating for reduced GR activity [16]. Heterozygous $f k b p 52$-deficient mice show increased susceptibility to high fat diet-induced hyperglycemia and hyperinsulinemia that correlates with reduced insulin clearance, hepatic steatosis and glucocorticoid resistance. This appears to be the outcome of reduced GR control of gluconeogenesis [17]. 
Much effort has gone into understanding the functional differences that distinguish FKBP52 from FKBP51, a closely related protein that contains approximately $70 \%$ similarity to FKBP52. fkbp51-deficient mice have also been generated and were initially observed to display no overt phenotypes. Interestingly knockout of both FKBP51 and FKBP51 results in an embryonic lethality phenotype (unpublished observations), although the cause of this phenotype has not been investigated. This finding demonstrates that FKBP51 and FKBP52 have some functional redundancies in physiology. Whether or not this functional redundancy is directly related to steroid hormone receptor regulation is unknown and studies to characterize the embryonic lethality phenotype are needed to answer this question.

\section{A Role for FKBP52 in Receptor Localization}

A given steroid receptor can be primarily cytoplasmic or nuclear, but it is not confined to a subcellular compartment. Actually, receptors undergo dynamic nucleo-cytoplasmic shuttling $(25,32)$, and ligand-binding displaces such equilibrium towards the nucleus. A debate exists regarding whether the Hsp90-based chaperone complex dissociates in the cytoplasm (a process referred to as 'transformation') or enters the nucleus still associated with the receptor. The traditional model posited the notion that transformation occurs rapidly upon ligand-binding, so the receptor exposes its nuclear localization signal (NLS) and concentrates in the nucleus by simple diffusion $[18,19]$. Nonetheless, recent evidence showed that dynein motor proteins associate with both the GR-Hsp90 and MR-Hsp90 complexes via FKBP52, suggesting that an active mechanism of retrotransport could take place. Both FKBP52 and dynein are recruited by the receptor heterocomplex upon steroid binding, whereas FKBP51, an immunophilin that binds inefficiently to dynein, is released [20]. The disruption of Hsp90 function, dynein binding or its motor function increased the nuclear translocation half-time for GR and MR by ten-fold [21,22]. Nevertheless, these receptors reach the nucleus, suggesting the existence of an alternative, Hsp90-FKBP52independent mechanism of movement (diffusion?). Inasmuch as the Hsp90-FKBP52 complex is essential for the efficient retrograde movement of the receptor, the next question is where transformation takes place.

The receptor must pass through the nuclear pore complex (NPC). This supramolecular structure permits the free diffusion of small $(<40 \mathrm{kDa})$ molecules. Larger compounds necessitate active transport, requiring interaction with importin $\alpha(\operatorname{Imp} \alpha)$, importin $\beta(\operatorname{Imp} \beta)$ and nuclear pore structures such as nucleoporins. Imp $\alpha$ binds the substrates' NLS and is complexed with $\operatorname{Imp} \beta$. The traditional model states that the NLS of steroid receptors remains hidden as long as Hsp90 is bound, and becomes exposed when the heterocomplex dissociates upon steroid binding. This model has come under question since there is persuasive evidence that an untransformed complex is not only required for GR association with the NPC, but also for an efficient passage through the pore [reviewed in 23]. Moreover, $\operatorname{Imp} \beta$ and Imp $\alpha$ have been recovered in association with GR-Hsp90 complexes [21]. Additionally intact MR-Hsp90-FKBP52 heterocomplexes have been recovered from the soluble portion of the nucleus [22]. $\operatorname{Imp} \beta$ and nucleoporins were also able to interact directly with GR. Finally, both cross-linked GR and MR accumulate in a steroid-dependent manner in the nucleus of digitonin-permeabilized cells [21,22]. While preincubation of permeabilized cells with NLS peptide or anti-NLS antibody fully inhibited the nuclear translocation of NLS-tagged albumin, neither treatment completely blocked MR nuclear translocation, suggesting again the possible existence of alternative mechanism of translocation [24]. In both cases, cytoplasmic transport and nuclear translocation, such an alternative system is always less efficient and slower than the Hsp90-FKBP52-dependent mechanism. This fact must have physiological consequences when the stimulus is deleterious (e.g. stress) and the homeostatic responses must be rapid. 
Interestingly, FKBP52 colocalizes with GR in the same nuclear speckles attached to the nuclear matrix. Treatment with high ionic strength and detergents cannot dissociate these speckles in wild-type fibroblasts, but GR is extracted in FKP52-KO cells (unpublished observations), suggesting that FKBP52 links GR to nuclear structures. The functional meaning of this observation is still unknown. One tempting speculation is that these speckles may work as "maturation centers" for post-translational modifications of GR

(phosphorylation, acetylation, sumoylation, etc.). Whatever the function is, the experimental evidence clearly shows that the propensity of FKBP52 to affect the properties of steroid receptors is not confined to the cytoplasmic complexes.

\section{The FKBP52 FK1 Proline-Rich Loop as an Interaction Surface}

Studies using chimeric receptor proteins in which the domains of ER and GR were switched localized FKBP52 regulation of GR function to the receptor ligand-binding domain (LBD) [3]. Given that FKBP52 contains a functional PPIase active site, the logical assumption is that FKBP52 associates with receptor-Hsp90 complexes and isomerizes a relevant proline substrate in the receptor LBD, thereby priming the receptor for hormone binding. However, mutations within the PPIase pocket that abolished PPIase activity failed to abrogate FKBP52 regulation of receptor function. Studies conducted by Riggs et al demonstrated that the FK1 domain itself is functionally important rather than the PPIase activity [7]. In particular, random mutagenesis was used to identify the proline-rich loop (Figure 1) overhanging the PPIase active site as being functionally important. Just two single point mutations (A116V and L119P) in the analogous loop on FKBP51, a highly similar protein that lacks receptorregulating ability in these assay systems, resulted in a gain-of-function in which mutant FKBP51 regulated receptor activity in a similar manner to that of FKBP52. These data suggest that differences in the proline-rich loop functionally distinguish FKBP51 and FKBP52 and that the proline-rich loop is functionally critical for the regulation of receptor activity. The current prevailing hypothesis is that the FKBP52 FK1 domain, the proline-rich loop in particular, comprises a functionally important interaction surface. This is an idea for which precedence exists as a co-crystallographic structure of a related family member, FKBP12, bound to transforming growth factor beta (TGF- $\beta$ ) demonstrates a direct interaction between the FKBP12 proline-rich loop and TGF- $\beta$ [25]. Interestingly, the proline residue analogous to proline 119 in FKBP52 participates directly in that interaction.

If the FKBP52 FK1 domain and the proline rich loop comprise an interaction surface, what is the interaction partner within the receptor-Hsp90 complex? Figure 2 illustrates known and predicted interactions between receptor, Hsp90 and FKBP52. FKBP52 is known to interact with the C-terminal EEVD motif on Hsp90 by way of the TPR domain [26,27]. The fact that FKBP52 regulation of receptor has been localized to the receptor LBD and that FKBP52 regulation is receptor-specific suggests that the interaction partner is the LBD. Thus, it is hypothesized that Hsp90 brings the FKBP52 FK1 domain in close proximity to the LBD and the proline-rich loop interacts with or at least transiently contacts the receptor. A single point mutation in the AR LBD, P723S, results in a mutant AR with increased dependence on FKBP52 for function (also termed FKBP52 hypersensitivity) [2]. In addition, our laboratory has identified additional mutations that confer a similar phenotype (unpublished observation). If these residues are viewed as a whole on the AR LBD crystal structure, they delineate a surface region that is within the recently characterized BF3 surface [28] on AR. Based on this observation, the AR BF3 surface is hypothesized to be a putative FKBP52 regulatory and/or interaction surface. 


\section{Therapeutically Targeting FKBP52}

The receptor specificity displayed by FKBP52 and the receptor-specific phenotypes observed in the fkbp52-deficient mice have established FKBP52 as an attractive therapeutic target for the treatment of hormone-dependent diseases. Given the receptor-specific phenotypes observed in the mice one would predict a role for FKBP52 in androgendependent diseases; most notably prostate cancer. Indeed, FKBP52 is up-regulated in prostate needle biopsies [29] and our early FKBP52-specific drug candidates have shown great promise at inhibiting prostate cancer cell proliferation and androgen-dependent gene expression (unpublished observations).

Given the role of FKBP52 in reproductive development and success $[2,4,10,12]$ FKBP52 could serve as a target for contraceptive drugs. FKBP52 is critical for embryo implantation in females [12-13] and could reasonably serve as a target for female contraception. Although fkbp52-deficient male mice have normal testis that produce viable sperm [2], the sperm do have reduced motility [11]. It is not clear if the testis in the mice have high enough testosterone levels produced locally that compensate for reduced AR activity in the absence of FKBP52, or if there is some factor present in the testis that can compensate for the loss of FKBP52. Thus, more studies to resolve these issues are needed, but FKBP52 may also serve as a target for male contraceptives.

Although FKBP52 can be found in association with estrogen receptor (ER)-chaperone complexes, an FKBP52-mediated functional effect on ER signaling has not been observed in cellular assays. However, FKBP52 is over-expressed in breast tumors [30] and is upregulated transcriptionally and post-transcriptionally by estrogen [31]. Exposure to the pure estrogen antagonist ICI182,780 (fulvestrant) prevents this estrogen-mediated increase. A recent study has found that the FKBP52 gene is methylated in ER-negative MDA-MB-231, but not in ER-positive MCF7 cells, suggesting that repression of FKBP52 may, itself, affect ER expression [32]. Thus, early studies implicate FKBP52 as a potential target for breast cancer, although more investigations into the role of FKBP52 in breast cancer are needed.

The current drug and drug candidates targeting receptor regulatory proteins lack sufficient specificity, and drugs targeting the receptor hormone binding pockets often lack efficacy in late stage disease. Figure 2 illustrates known and predicted interactions in addition to known and possible therapeutic targeting strategies. Geldanamycin and its analogs, for example, exhibit potent antitumor activity by inhibiting $\mathrm{Hsp} 90$ interaction with client proteins, leading to increased client protein degradation. However, these drugs could potentially affect any Hsp90 client protein leading to adverse side effects, although the Hsp90 inhibitors do preferentially target cancer cells [reviewed in 33]. In addition, geldanamycin has been completely ineffective in prostate cancer.

Immunophilins are targets of the immunosuppressant macrolide FK506 (Tacrolimus), which is clinically used after organ transplantation [review in 34]. FK506 is a large molecule that binds the PPIase pocket and protrudes out, forming a false interaction surface that binds with high affinity to calcineurin resulting in immunosuppression [35]. FK506 does not discriminate among the FKBP family members and immunosuppression is not a desirable side effect for an anti-cancer drug. Although FK506 binding to FKBP52 is not immunosuppressive, it does inhibit FKBP52-mediated potentiation of steroid receptor function and inhibits prostate cancer cell proliferation [36]. This not only demonstrates that FKBP52 is a "druggable" protein, but also provides proof of principle for the targeting of FKBP52 for the treatment of prostate cancer. FK506 could be used as a scaffold to design a large molecule that protrudes out of the PPIase pocket and interferes with FKBP52 prolinerich loop interactions, but that does not share the parent compound's affinity for calcineunin 
and its immunosuppressive effect. However, such a molecule may not be able to discriminate between the FKBPs given the conservation of the PPIase pocket. Considering the highly conserved nature of the TPR domain, especially among the FKBP family members, there are also concerns about targeting the FKBP52 TPR domain as well. The recent work by Riggs et al [7] suggests that blocking proline-rich loop interactions is potentially a highly specific therapeutic strategy to inhibit receptor function. It is likely that small molecule inhibitors specific for the FKBP52 FK1 domain, and perhaps even the proline-rich loop, can be developed. Finally, threonine 143 within the FKBP52 FK Linker (Figure 1) that links the FK1 and FK2 domains is phosphorylated by casein kinase 2 and this phosphorylation event was demonstrated to reorient the entire FK1 domain leading to a loss of FKBP52 function $[37,38]$. Thus, targeting the FKBP52 FK Linker may be a viable option. Our laboratory is currently pursuing multiple strategies for the development of FKBP52specific inhibitors.

\section{Conclusion}

FKBP52 has emerged as an extremely attractive therapeutic target. While much has been learned regarding the role of FKBP52 in physiology, we still do not fully understand how FKBP52 regulates receptors from a structural and mechanistic point of view. Structural and biochemical studies to understand the manner in which FKBP52 interacts within the receptor-Hsp90 complex (e.g. cryo-EM and/or co-crystal structures) and what the interaction partners are (e.g. cross-linking and peptide mapping) are needed. While efforts are underway to target FKBP52 with small molecule inhibitors, these types of studies would greatly enhance these efforts. Finally, there is mounting evidence that FKBP52 not only directly participates in receptor association with the nuclear pore and nuclear translocation, but also has a continued role in regulating receptor function once in the nucleus. Thus, studies that distinguish between the cytoplasmic and nuclear roles of FKBP52 are also needed.

\section{Acknowledgments}

The authors are in part supported by the Grant Number 5G12RR008124 (to the Border Biomedical Research Center (BBRC)/University of Texas at El Paso) from the National Center for Research Resources (NCRR), a component of the National Institutes of Health (NIH). Its contents are solely the responsibility of the authors and do not necessarily represent the official views of NCRR or NIH. We thank the Border Biomedical Research Center's Biomolecule Analysis Core Facility (BACF), Tissue Culture Core Facility (TCF), and the DNA Analysis Core Facility (DACF) for the use of the instruments. M.B.C. is also supported in part by American Recovery and Reinvestment Act (ARRA) funds through grant number SC1GM084863 from the National Institute of General Medical Sciences, NIH. M.D.G. is supported by grant UBACyT-X085 from the University of Buenos Aires.

\section{References and recommended reading}

Papers of particular interest, published within the period of review, have been highlighted as:

- of special interest

•• of outstanding interest

1. Smith DF, Toft DO. Minireview: the intersection of steroid receptors with molecular chaperones: observations and questions. Mol Endocrinol. 2008; 22:2229-2240. [PubMed: 18451092]

2 ••. Cheung-Flynn J, Prapapanich V, Cox MB, Riggs DL, Suarez-Quian C, Smith DF. Physiological role for the cochaperone FKBP52 in androgen receptor signaling. Mol Endocrinol. 2005; 19:1654-1666. See annotation to Ref. 17. In addition, this study introduced the AR P723S mutant which displays hypersensitivity to FKBP52 regulation. This result helped establish the AR BF3 surface as a putative FKBP52 regulatory and/or interaction surface. [PubMed: 15831525] 
$3 \bullet$ Riggs DL, Roberts PJ, Chirillo SC, Cheung-Flynn J, Prapapanich V, Ratajczak T, Gaber R, Picard D, Smith DF. The Hsp90-binding peptidylprolyl isomerase FKBP52 potentiates glucocorticoid signaling in vivo. EMBO J. 2003; 22:1158-1167. This study localized FKBP52 regulation of receptor function to the receptor hormone binding domain. [PubMed: 12606580]

4 ••. Tranguch S, Cheung-Flynn J, Daikoku T, Prapapanich V, Cox MB, Xie H, Wang H, Das SK, Smith DF, Dey SK. Cochaperone immunophilin FKBP52 is critical to uterine receptivity for embryo implantation. Proc Natl Acad Sci U S A. 2005; 102:14326-14331. See annotation to Ref. 17. [PubMed: 16176985]

5. Riggs DL, Cox MB, Cheung-Flynn J, Prapapanich V, Carrigan PE, Smith DF. Functional specificity of co-chaperone interactions with Hsp90 client proteins. Crit Rev Biochem Mol Biol. 2004; 39:279295. [PubMed: 15763706]

6. Davies TH, Ning YM, Sanchez ER. Differential control of glucocorticoid receptor hormone-binding function by tetratricopeptide repeat (TPR) proteins and the immunosuppressive ligand FK506. Biochemistry. 2005; 44:2030-2038. [PubMed: 15697228]

7 •. Riggs DL, Cox MB, Tardif HL, Hessling M, Buchner J, Smith DF. Noncatalytic role of the FKBP52 peptidyl-prolyl isomerase domain in the regulation of steroid hormone signaling. Mol Cell Biol. 2007; 27:8658-8669. This study firmly established the FKBP52 FK1 domain and the proline-rich loop as a critically important functional domain that likely serves as an interaction surface. [PubMed: 17938211]

8. Echeverria PC, Picard D. Molecular chaperones, essential partners of steroid hormone receptors for activity and mobility. Biochim Biophys Acta. 2010; 1803:641-649. [PubMed: 20006655]

9. Wu B, Li P, Liu Y, Lou Z, Ding Y, Shu C, Ye S, Bartlam M, Shen B, Rao Z. 3D structure of human FK506-binding protein 52: Implications for the assembly of the glucocorticoid receptor/Hsp90/ immunophilin heterocomplex. Proc Natl Acad Sci U S A. 2004; 101:8348-8353. [PubMed: 15159550]

$10 \bullet$ • Yong W, Yang Z, Periyasamy S, Chen H, Yucel S, Li W, Lin LY, Wolf IM, Cohn MJ, Baskin LS, et al. Essential role for Co-chaperone Fkbp52 but not Fkbp51 in androgen receptor-mediated signaling and physiology. J Biol Chem. 2007; 282:5026-5036. See annotation to Ref. 17. [PubMed: 17142810]

$11 \bullet$. Hong J, Kim ST, Tranguch S, Smith DF, Dey SK. Deficiency of co-chaperone immunophilin FKBP52 compromises sperm fertilizing capacity. Reproduction. 2007; 133:395-403. See annotation to Ref. 17. [PubMed: 17307907]

12 ••. Tranguch S, Wang H, Daikoku T, Xie H, Smith DF, Dey SK. FKBP52 deficiency-conferred uterine progesterone resistance is genetic background and pregnancy stage specific. J Clin Invest. 2007; 117:1824-1834. See annotation to Ref. 17. [PubMed: 17571166]

13 ••. Yang Z, Wolf IM, Chen H, Periyasamy S, Chen Z, Yong W, Shi S, Zhao W, Xu J, Srivastava A, et al. FK506-binding protein 52 is essential to uterine reproductive physiology controlled by the progesterone receptor A isoform. Mol Endocrinol. 2006; 20:2682-2694. See annotation to Ref. 17. [PubMed: 16873445]

14. Hirota Y, Acar N, Tranguch S, Burnum KE, Xie H, Kodama A, Osuga Y, Ustunel I, Friedman DB, Caprioli RM, et al. Uterine FK506-binding protein 52 (FKBP52)-peroxiredoxin-6 (PRDX6) signaling protects pregnancy from overt oxidative stress. Proc Natl Acad Sci U S A. 2010; 107:15577-15582. [PubMed: 20713718]

$15 \bullet$. Hirota Y, Tranguch S, Daikoku T, Hasegawa A, Osuga Y, Taketani Y, Dey SK. Deficiency of immunophilin FKBP52 promotes endometriosis. Am J Pathol. 2008; 173:1747-1757. See annotation to Ref. 17. [PubMed: 18988805]

16. Cox, MB.; Smith, DF. Eurekah.com. Functions of the Hsp90-Binding FKBP Immunophilins. In: Blatch, GL., editor. The Networking of Chaperones by Cochaperones. 2006.

17 ••. Warrier M, Hinds TD Jr, Ledford KJ, Cash HA, Patel PR, Bowman TA, Stechschulte LA, Yong W, Shou W, Najjar SM, et al. Susceptibility to diet-induced hepatic steatosis and glucocorticoid resistance in FK506-binding protein 52-deficient mice. Endocrinology. 2010; 151:3225-3236. This study in addition to Refs. [2, 4, 10-13, and 15] details the AR, GR, and PR-related phenotypes that have been characterized in the fkbp52-deficient mice. These receptor-specific phenotypes have collectively established FKBP52 as a promising drug target for the treatment of a variety of hormone-dependent diseases. [PubMed: 20427484] 
18. Gorlich D, Kutay U. Transport between the cell nucleus and the cytoplasm. Annu Rev Cell Dev Biol. 1999; 15:607-660. [PubMed: 10611974]

19. Stewart M. Molecular mechanism of the nuclear protein import cycle. Nat Rev Mol Cell Biol. 2007; 8:195-208. [PubMed: 17287812]

20. Wochnik GM, Ruegg J, Abel GA, Schmidt U, Holsboer F, Rein T. FK506-binding proteins 51 and 52 differentially regulate dynein interaction and nuclear translocation of the glucocorticoid receptor in mammalian cells. J Biol Chem. 2005; 280:4609-4616. [PubMed: 15591061]

21 •. Echeverria PC, Mazaira G, Erlejman A, Gomez-Sanchez C, Piwien Pilipuk G, Galigniana MD. Nuclear import of the glucocorticoid receptor-hsp90 complex through the nuclear pore complex is mediated by its interaction with Nup62 and importin beta. Mol Cell Biol. 2009; 29:4788-4797. See annotation to Ref. 22. [PubMed: 19581287]

22 •• Galigniana MD, Erlejman AG, Monte M, Gomez-Sanchez C, Piwien-Pilipuk G. The hsp90FKBP52 complex links the mineralocorticoid receptor to motor proteins and persists bound to the receptor in early nuclear events. Mol Cell Biol. 2010; 30:1285-1298. This study in addition to Ref. 21 provides compelling evidence that Hsp90 and FKBP52 are not only required for receptor association with the nuclear pore and nuclear import but also demonstrates that $\mathrm{Hsp} 90$ and FKBP52 remain associated with receptor inside the nucleus. [PubMed: 20038533]

23. Galigniana MD, Echeverria PC, Erlejman AG, Piwien-Pilipuk G. Role of molecular chaperones and TPR-domain proteins in the cytoplasmic transport of steroid receptors and their passage through the nuclear pore. Nucleus. 2010; 1:299-308. [PubMed: 21113270]

24. Piwien Pilipuk G, Vinson GP, Sanchez CG, Galigniana MD. Evidence for NL1-independent nuclear translocation of the mineralocorticoid receptor. Biochemistry. 2007; 46:1389-1397. [PubMed: 17260968]

25. Huse M, Chen YG, Massague J, Kuriyan J. Crystal structure of the cytoplasmic domain of the type I TGF beta receptor in complex with FKBP12. Cell. 1999; 96:425-436. [PubMed: 10025408]

26. Cheung-Flynn J, Roberts PJ, Riggs DL, Smith DF. C-terminal Sequences outside the Tetratricopeptide Repeat Domain of FKBP51 and FKBP52 Cause Differential Binding to Hsp90. J Biol Chem. 2003; 278:17388-17394. [PubMed: 12611898]

27. Smith DF. Tetratricopeptide repeat cochaperones in steroid receptor complexes. Cell Stress Chap. 2004; 9:109-121.

28 • Estebanez-Perpina E, Arnold LA, Nguyen P, Rodrigues ED, Mar E, Bateman R, Pallai P, Shokat KM, Baxter JD, Guy RK, et al. A surface on the androgen receptor that allosterically regulates coactivator binding. Proc Natl Acad Sci U S A. 2007; 104:16074-16079. This is the first study to characterize the AR BF3 surface which is proposed to be the FKBP52 regulatory and/or interaction surface on the receptor. [PubMed: 17911242]

29. Lin JF, Xu J, Tian HY, Gao X, Chen QX, Gu Q, Xu GJ, Song JD, Zhao FK. Identification of candidate prostate cancer biomarkers in prostate needle biopsy specimens using proteomic analysis. Int J Cancer. 2007; 121:2596-2605. [PubMed: 17722004]

30. Ward BK, Mark PJ, Ingram DM, Minchin RF, Ratajczak T. Expression of the estrogen receptorassociated immunophilins, cyclophilin 40 and FKBP52, in breast cancer. Breast Cancer Res Treat. 2000; 58:267-280. [PubMed: 10718488]

31. Kumar P, Mark PJ, Ward BK, Minchin RF, Ratajczak T. Estradiol-regulated expression of the immunophilins cyclophilin 40 and FKBP52 in MCF-7 breast cancer cells. Biochem Biophys Res Commun. 2001; 284:219-225. [PubMed: 11374893]

32. Ostrow KL, Park HL, Hoque MO, Kim MS, Liu J, Argani P, Westra W, Criekinge WV, Sidransky D. Pharmacologic unmasking of epigenetically silenced genes in breast cancer. Clin Cancer Res. 2009; 15:1184-1191. [PubMed: 19228724]

33. Trepel J, Mollapour M, Giaccone G, Neckers L. Targeting the dynamic HSP90 complex in cancer. Nat Rev Cancer. 2010; 10:537-549. [PubMed: 20651736]

34. Dumont FJ. FK506, an immunosuppressant targeting calcineurin function. Curr Med Chem. 2000; 7:731-748. [PubMed: 10702636]

35. Hamilton GS, Steiner JP. Immunophilins: beyond immunosuppression. J Med Chem. 1998; 41:5119-5143. [PubMed: 9857082] 
$36 \bullet$. Periyasamy S, Warrier M, Tillekeratne MP, Shou W, Sanchez ER. The immunophilin ligands cyclosporin A and FK506 suppress prostate cancer cell growth by androgen receptor-dependent and -independent mechanisms. Endocrinology. 2007; 148:4716-4726. This study establishes the targeting of FKBP proteins as a viable option for the treatment of prostate cancer. [PubMed: 17615153]

37. Cox MB, Riggs DL, Hessling M, Schumacher F, Buchner J, Smith DF. FK506-binding protein 52 phosphorylation: a potential mechanism for regulating steroid hormone receptor activity. Mol Endocrinol. 2007; 21:2956-2967. [PubMed: 17717070]

38. Miyata Y, Chambraud B, Radanyi C, Leclerc J, Lebeau MC, Renoir JM, Shirai R, Catelli MG, Yahara I, Baulieu EE. Phosphorylation of the immunosuppressant FK506-binding protein FKBP52 by casein kinase II: regulation of HSP90-binding activity of FKBP52. Proc Natl Acad Sci U S A. 1997; 94:14500-14505. [PubMed: 9405642] 


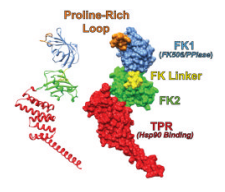

Figure 1. Three-Dimensional Structure for FKBP52

A composite of two partial structures for human FKBP52 (protein databank numbers 1Q1C and 1P5Q). The left panel illustrates the FKBP52 structure in ribbon format and the right panel in molecular surface format. The individual domains as well as regions of functional importance are individually colored. The TPR domain (red) allows association with Hsp90receptor complexes through interaction with the Hsp90 EEVD motif. The FK1 domain (blue) contains the PPIase active site as well as the proline-rich loop (orange), which is proposed to serve as an interaction surface. FK1 is linked to the FK2 domain (green) by way of the FK Linker (yellow). FK2, although similar to FK1, lacks PPIase activity. The FK Linker contains a casein kinase 2 phosphorylation site that, when phosphorylated, abrogates FKBP52 function. The two partial structures were overlayed and the figure was created using UCSF Chimera version 1.5. 


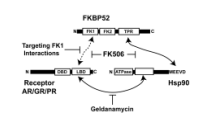

Figure 2. FKBP52 Interactions and Targeting Strategies

Interactions: Known (solid arrow) and predicted (dashed arrow) interactions between FKBP52, Hsp90 and the receptor are shown. FKBP52 is known to interact with the Cterminal EEVD on Hsp90 and Hsp90 interacts with the receptor ligand binding domain. Based on the available evidence it is hypothesized that Hsp90 brings the FKBP52 FK1 domain, the proline-rich loop in particular, in close proximity to the receptor LBD where FKBP52 at least contacts the receptor leading to regulation of receptor hormone binding and subcellular localization. Targeting the interactions: Several drugs and drug candidates are available for disrupting receptor-Hsp90 complexes. The Hsp90 inhibitor geldanamycin and derivatives bind to the $\mathrm{N}$-terminal nucleotide-binding site, which disrupts complex formation and leads to receptor degradation. However, this class of compounds lacks Hsp90 client protein specificity and has been ineffective in prostate cancer. The immunosuppressive ligand FK506 binds the FKBP52 PPIase pocket and is known to disrupt FKBP52 regulation of receptor. However, this drug lacks FKBP protein specificity and is highly immunosuppressive. If the FK1 domain and the proline-rich loop do serve as an interaction surface, then targeting those interactions is likely to be a viable option. 